\title{
Modulator property of the intrinsic cortical projection from layer 6 to layer 4
}

\section{Charles C. Lee* and S. Murray Sherman}

Department of Neurobiology, University of Chicago, Chicago, IL, USA

\section{Edited by:}

Mriganka Sur, Massachusetts Institute

of Technology (MIT), USA

Reviewed by:

Charles Cox, University of Illinois at

Urbana-Champaign, USA

Michael J. Friedlander, Baylor College

of Medicine, USA

*Correspondence:

Charles C. Lee, Department of

Neurobiology, University of Chicago,

947 E. 58th St, MC 0926, Chicago,

IL 60637, USA.

e-mail: clee@bsd.uchicago.edu
Layer 4 of the sensory neocortex receives widespread convergent inputs from thalamic, intracortical, and corticocortical sources. Yet, the relative information bearing roles for most of these pathways remain largely undefined. Here we show that the intracortical projections from layer 6 to layer 4 exhibit a physiological property that is consistent with a modulator role. Using in vitro slice preparations of the auditory and somatosensory cortices, we found that electrical stimulation or photostimulation of layer 6 elicits a prolonged depolarizing response that is attributable to the activation of group 1 metabotropic glutamate receptors. These results complement the known physiological properties of the layer 6 to layer 4 pathway, and further suggest that this pathway is not a principle conduit for information flow, but rather acts as a modulator of cortical activity.

Keywords: cortex, drivers, modulators, intracortical

\section{INTRODUCTION}

The sensory neocortex is composed of six layers whose distinct patterns of connectivity underlie their functional properties. A major challenge for understanding information processing in the sensory neocortex is to determine how information is transferred and transformed from the principal cortical input layers to the principal cortical output layers. In this regard, multiple inputs converge onto layer 4 neurons of the sensory neocortex from thalamic (Sherman and Guillery, 2002), intracortical (Hirsch and Martinez, 2006) and corticocortical (Rockland and Pandya, 1979) sources. Quantitative estimates suggest that the intrinsic cortical projections provide about half of this total convergent input, while inputs from thalamic sources are an order of magnitude less ( $\sim 5 \%$; Ahmed et al., 1994; Binzegger et al., 2004; Latawiec et al., 2000; Lee et al., 2004). In addition, previous work has demonstrated the importance of the thalamic inputs to layer 4 for information processing (Ferster et al., 1996; Lee and Sherman, 2008; Reid and Alonso, 1995; Sherman and Guillery, 2002), but the role of the other inputs, in particular those from intracortical layer 6 (Stratford et al., 1996; Tarczy Hornoch et al., 1999), remains less clear.

Assessing the information-bearing role of the intracortical pathway from layer 6 to layer 4 poses an important challenge (Raizada and Grossberg, 2003), but it is one that can be addressed partly using physiological metrics, as recently demonstrated (Bartlett and Smith, 2002; Li et al., 2003). Glutamatergic pathways can be parsed into two main categories, termed "drivers" and "modulators" (Lee and Sherman, 2008; Reichova and Sherman, 2004; Sherman and Guillery, 1998), and possibly others that have yet to be demonstrated (see Discussion). Driver pathways are the principal conduits for information flow, and exhibit synaptic properties such as a large all-or-none response, paired-pulse depression, and lack of a metabotropic glutamate receptor (mGluR) component to the response. In contrast, modulator pathways exhibit small, graded responses, paired-pulse facilitation, and activate mGluRs (Sherman and Guillery, 2005). In particular, the group 1 mGluRs have been found to correlate strongly with modulator synapses in the thalamus (Bartlett and Smith, 2002; Reichova and Sherman, 2004). It is this property that is of particular interest, since group $1 \mathrm{mGluRs}$ have been reported on layer 4 neurons in the sensory neocortex (LópezBendito et al., 2002; Ryo et al., 1993; Stinehelfer et al., 2000), and thus may be activated postsynaptically by the intracortical pathway from layer 6. Indeed, it is known that the thalamic inputs to layer 4, which exhibit driving synaptic properties, do not elicit a group 1 mGluR response, while the intracortical layer 6 inputs exhibit all of the other synaptic properties associated with modulator pathways (Lee and Sherman, 2008). Thus, determining whether the layer 6 inputs to layer 4 also elicit a group $1 \mathrm{mGluR}$ response would help further to characterize its role in information processing.

Therefore, we investigated whether this modulator property was present in the intracortical projection from layer 6 to layer 4 using in vitro slice preparations of the somatosensory and auditory cortices. Using both electrical stimulation and photostimulation with caged glutamate to activate the intracortical inputs from layer 6 to layer 4 , we found that a group $1 \mathrm{mGluR}$ component could be elicited. These results and their functional implications are described below.

\section{MATERIALS AND METHODS}

Slice preparations were made from mice (ages 10-16 days), as described in previous work (Lee and Sherman, 2008). All procedures were approved by the Institutional Animal Care and Use Committee of the University of Chicago. Briefly, mice were anesthetized with isoflurane then decapitated. Whole brains were quickly dissected and placed into cool, oxygenated, artificial cerebral spinal fluid (ACSF). A vibratome (Campden Instruments, Lafayette, IN, USA) was used to cut slices $(500 \mu \mathrm{m})$, which were then recovered in physiological ACSF (in mM: $125 \mathrm{NaCl}, 25 \mathrm{NaHCO}_{3}, 3 \mathrm{KCl}, 1.25$ $\mathrm{NaH}_{2} \mathrm{PO}_{4}, 1 \mathrm{MgCl}_{2}, 2 \mathrm{CaCl}_{2}, 25$ glucose) for $1 \mathrm{~h}$ at $32^{\circ} \mathrm{C}$. Slices were then placed in a submersion-type recording chamber on a modified microscope stage, and maintained at $32^{\circ} \mathrm{C}$ with constant perfusion of ACSF. 
DIC optics was used to identify the cortical sites for recording and stimulation. Whole cell recordings were made with pipettes containing intracellular solution (135 KGluconate, $7 \mathrm{NaCl}, 10$ HEPES, $1-2 \mathrm{Na}_{2}$ ATP, $0.3 \mathrm{GTP}, 2 \mathrm{MgCl}_{2}$ and $0.5 \%$ biocytin at a $\mathrm{pH}$ of 7.3 obtained with $\mathrm{KOH}$ and osmolality of 290 mosm obtained with distilled water). Current or voltage clamp recordings were made using the Axoclamp 2A amplifier and pCLAMP software (Axon Instruments, Union City, CA, USA), and were uncorrected for junction potentials $(\sim 10 \mathrm{mV})$. Depolarizing current injections were used to determine the spiking characteristics of layer 4 neurons. Neurons were classified as regular spiking (RS) if they fired at slow adapting frequencies with small and slow afterhyperpolarizations (AHPs), in comparison to fast spiking (FS) neurons that had higher maximal firing rates, with large and fast AHPs (Lee and Sherman, 2008). The acquired data were digitized using a Digidata 1200 board and then stored in a computer for later analysis.

Receptor antagonists were prepared in distilled water, diluted to their final concentration just before use, and then bath applied. The final bath concentration was generally estimated to be onefourth of the initial concentration based on the rate of injection. To block ionotropic GluRs during high-frequency stimulation (HFS), DNQX $(50 \mu \mathrm{M})$ for AMPA and MK-801 $(40 \mu \mathrm{M})$ for NMDA were used. To block GABA receptors, SR 95531 (50 nM) for $\mathrm{GABA}_{\mathrm{A}}$ and CGP 46381 ( $50 \mathrm{nM}$ ) for $\mathrm{GABA}_{\mathrm{B}}$ were used. To block the group 1 mGluRs, LY367385 $(20 \mu \mathrm{M})$ for mGluR1 and MPEP $(30 \mu \mathrm{M})$ for mGluR5 (TOCRIS, Ellisville, MO, USA) were added together to the bath. To block the group 2 mGluRs, MCCG $(50 \mu \mathrm{M})$ was used.

Electrical stimulation of the layer 6 inputs to layer 4 was performed using bipolar concentric electrodes (Frederick Haer, Bowdoinham,
ME, USA). Stimulation consisted of pulses of $0.1-0.2 \mathrm{~ms}$ delivered using an electrical stimulus protocol. Photostimulation was used to identify the layer 6 regions projecting to layer 4 , and the stimulation electrode was situated in the layer 6 hotspot that produced the most robust responses with the lowest stimulus intensity. HFS consisted of pulses at $125 \mathrm{~Hz}$ for $800 \mathrm{~ms}$. Amplitudes of evoked responses were measured at the peak of the response. During application of group $1 \mathrm{mGluR}$ antagonists, amplitudes were measured at the same time to peak from the control condition.

For photostimulation, nitroindolinyl (NI)-caged glutamate (0.37 mM; Sigma-RBI) was added to the recirculating ACSF (Lam and Sherman, 2007; Shepherd et al., 2003). Photolysis of the caged glutamate was done focally with an average beam intensity of $5 \mathrm{~mW}$ to give a 1-msec, 100-pulse light stimulus by Q-switching the pulsed UV laser (355 nm wavelength, frequency-tripled $\mathrm{Nd}$ : YVO4, $100 \mathrm{kHz}$ pulse repetition rate; DPSS Lasers, Inc., Santa Clara, CA, USA). Custom software written in MATLAB (MathWorks, Inc., Natick, MA, USA) was used to analyze the data, and the acquired traces were superimposed on a photomicrograph corresponding to the stimulation sites.

\section{RESULTS}

In order to examine the properties of the intracortical synapse from layer 6 to layer 4, we utilized in vitro slice preparations containing the mouse somatosensory and auditory cortices, as described previously (Lee and Sherman, 2008). Whole-cell, current-clamp recordings were obtained from layer 4 neurons in response to electrical stimulation or photostimulation of layer 6 (Figures 1-2). A total of 21 neurons (RS: $n=16$; FS: $n=5$ ) were recorded, with
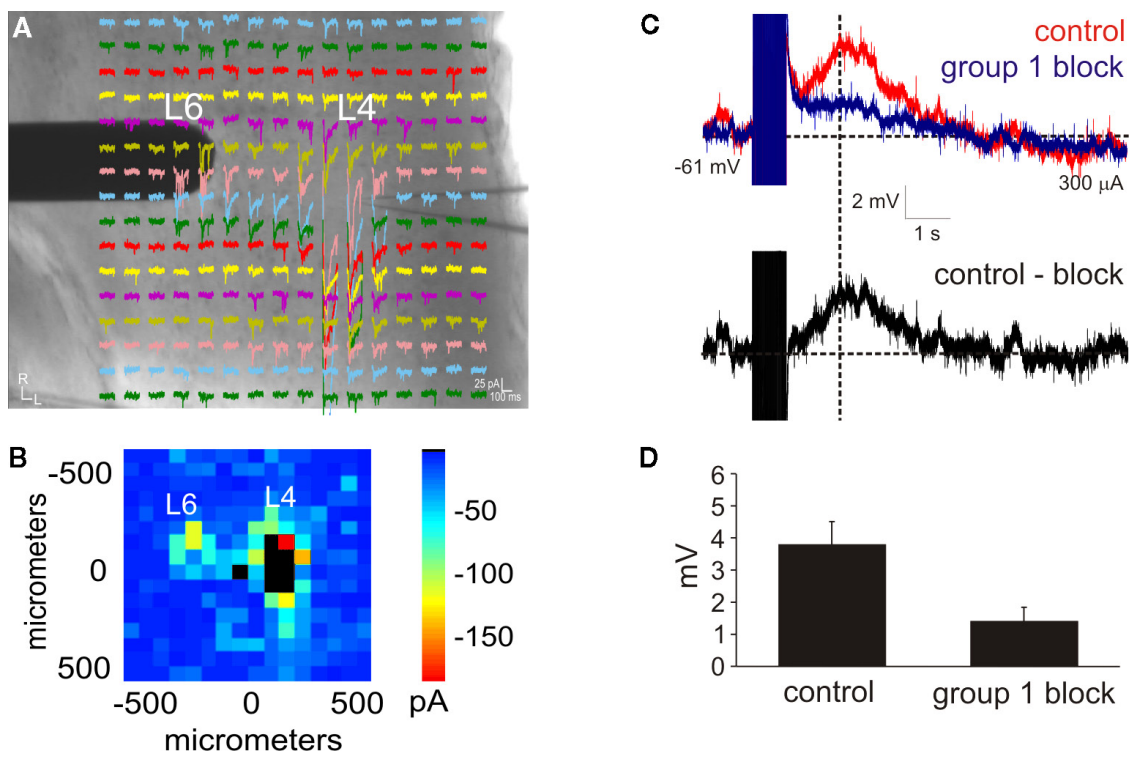

D

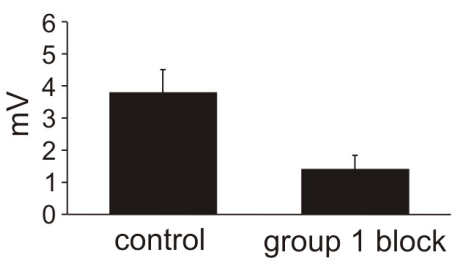

FIGURE 1 | Intracortical projections from layer 6 to layer 4 exhibit a modulator property. $(\mathbf{A}, \mathbf{B})$ Photostimulation with caged glutamate in the cortex (All) identifies the location of layer 6 input to layer 4. (A) Whole-cell, voltage clamp recordings from layer $4(\llcorner 4)$ in response to photostimulation at 256 separate cortical loci (colored traces). A concentric bipolar electrode was targeted to the region in layer 6 (L6) that elicited the strongest photostimulation response. (B) Response map plot illustrating the peak EPSC amplitude within $100 \mathrm{~ms}$ of the stimulus onset. (C) Whole-cell, current clamp recordings in

layer 4 following high-frequency electrical stimulation in layer 6. A large depolarizing response is elicited after blocking iGluRs, GABARs, and group 2 mGluRs (red trace). Further blocking of the group 1 mGluRs abolishes the response (blue trace). The group $1 \mathrm{mGluR}$ response is isolated in a subtracted trace (control-block: black trace). Baselines denoted by horizontal dashed lines. Amplitudes were measured at the peak time denoted by the vertical dash line. (D) The amplitude of the depolarizing response is significantly reduced in the presence of group $1 \mathrm{mGluR}$ antagonists ( $p<0.05$, $t$-test). 

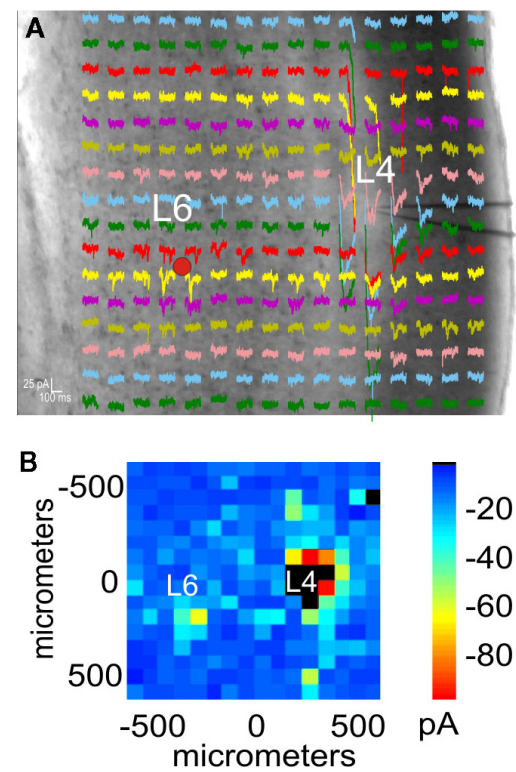

FIGURE 2 | Photostimulation of the layer 6 input to layer 4. (A,B) Photostimulation with caged glutamate in the cortex (All) identifies the location of layer 6 input to layer 4. (A) Whole-cell, voltage clamp recordings from layer 4 (L4) in response to photostimulation at 256 separate cortical loci (colored traces). (B) Response map plot illustrating the peak EPSC amplitude within $100 \mathrm{~ms}$ of the stimulus onset. (C) After pharmacologically blocking receptors for GABA ${ }_{A^{\prime}} \mathrm{GABA}_{\mathrm{B}^{\prime}}$ group $2 \mathrm{mGluR}$, and NMDA and photostimulating in layer 6 (red dot in A), a large AMPA response is elicited

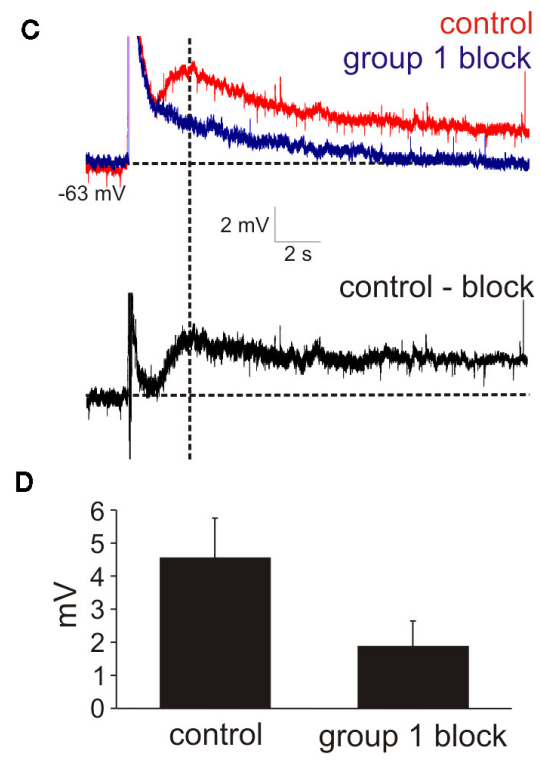

followed by a slow depolarizing response (red trace). Blocking the group 1 metabotropic glutamate receptors abolishes the slow depolarizing response, leaving the AMPA mediated response (blue trace). The group 1 mGluR response is observed in a subtracted trace (control-block: black trace). Baselines denoted by horizontal dashed lines. Amplitudes were measured at the peak time denoted by the vertical dash line. (D) In the presence of group $1 \mathrm{mGluR}$ antagonists, the amplitude of the depolarizing response is significantly reduced $(p<0.05, t$-test). an average resting potential of $-63.1 \pm 4.8 \mathrm{mV}$ and input resistance of $216.9 \pm 81.3 \mathrm{M} \Omega$.

An important synaptic property of modulatory glutamatergic synapses is the presence of postsynaptic group 1 mGluRs (Reichova and Sherman, 2004). Thus, we physiologically tested whether the intracortical pathway from layer 6 to layer 4 (Stratford et al., 1996) can activate a group $1 \mathrm{mGluR}$ response. To identify the precise location of the layer 6 inputs, photostimulation with caged glutamate (Lam and Sherman, 2007; Shepherd et al., 2003) was used to first map the intracortical inputs to layer 4 (Figure 1A) in a total of 12 neurons from somatosensory (SI: $n=3$; SII: $n=3$ ) and auditory (AI: $n=3$; AII: $n=3$ ) cortical areas. Subsequently, a stimulating electrode was targeted to the layer 6 hotspot as identified by photostimulation in order to electrically activate the mGluR response (Figure 1A), and was also used in related experiments to test for paired-pulse effects (see Figure 7 in Lee and Sherman, 2008). In order to isolate the response of group $1 \mathrm{mGluRs}$, iGluRs were pharmacologically blocked with DNQX (for AMPARs) and MK-801 (for NMDARs), GABARs were blocked with SR 95531 (for GABA $_{A}$ Rs) and CGP $46381\left(\mathrm{GABA}_{\mathrm{B}} \mathrm{Rs}\right)$, and group $2 \mathrm{mGluRs}$ were blocked with MCCG (Lee and Sherman, 2009). To evoke the mGluR response, HFS was then applied ( $125 \mathrm{~Hz}$ for $800 \mathrm{~ms}$ ) (McCormick and Von Krosigk, 1992; Reichova and Sherman, 2004). In all of the tested neurons, HFS elicited a large and long lasting depolarizing response (peak amplitude: $3.8 \pm 0.8 \mathrm{mV}$; Figure $1 \mathrm{C}$, red trace). Following the addition of group $1 \mathrm{mGluR}$ antagonists (LY367385 for mGluR1 and MPEP for mGluR5; Figure 1C, blue trace), the amplitude of the depolarization, measured at the same peak time from the control condition, was significantly decreased $(1.6 \pm 0.5 \mathrm{mV} ; p<0.05$, $t$-test; Figure 1D). The group 1 response alone could be observed in a subtracted trace (Figure 1C, black trace). Thus, electrical stimulation of the layer 6 to layer 4 pathway is capable of eliciting a modulator response mediated by group 1 mGluRs.

Since electrical stimulation may activate fibers of passage, we sought to test if this physiological property could be elicited solely with photostimulation, which only activates neurons at the cell body or proximal dendrites (Lam and Sherman, 2007; Shepherd et al., 2003). In a total of nine neurons (SI: $n=2$; SII: $n=3$; AI: $n=2$; AII: $n=2$ ), the layer 6 region projecting to layer 4 was identified via photostimulation (Figures 2A,B). Receptor antagonists for $G_{A B A}, G A B A_{B}$, group 2 mGluRs, and NMDA were then added to the recirculating bath, and subsequent photostimulation of the layer 6 inputs to layer 4 elicited a fast AMPA response followed by a large, slow depolarizing response (peak amplitude: $4.6 \pm 1.1 \mathrm{mV}$; Figure $2 \mathrm{C}$, red trace). The amplitude of this slow depolarizing response, measured at the same peak time from the control condition, was significantly reduced ( $1.8 \pm 0.8 \mathrm{mV} ; p<0.05$, $t$-test; Figure 2D) in the presence of group $1 \mathrm{mGluR}$ antagonists (Figure 2C, blue trace). A subtracted trace demonstrated the isolated group 1 response (Figure 2C, black trace). Thus, photostimulation of the layer 6 to layer 4 pathway is also capable of eliciting the modulator response mediated by group 1 mGluRs.

\section{DISCUSSION}

Cortical neurons in layer 4 have previously been shown to express group 1 mGluRs (López-Bendito et al., 2002; Ryo et al., 1993; 


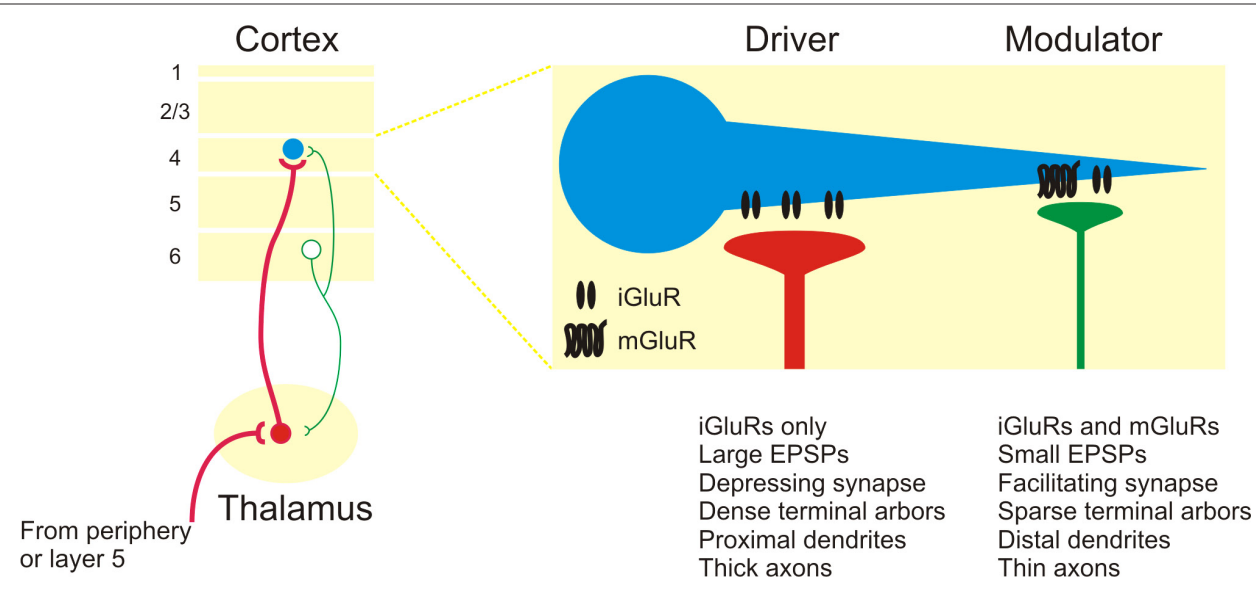

FIGURE 3 | Summary of driver and modulator inputs to layer 4 neurons in sensory neocortex. Driver inputs (red lines) to layer 4 neurons (blue circles) originate primarily from thalamic sources, while modulator inputs (green lines) originate from the intracortical layer 6 projection. Among the many distinctions, modulator inputs activate both iGluRs and mGluRs, while driver inputs only activate iGluRs.
Stinehelfer et al., 2000). However, it was largely unclear which forebrain pathways activated these receptors. Our results extend these previous anatomical findings by demonstrating that the intracortical projections from layer 6 are capable of postsynaptically eliciting the group $1 \mathrm{mGluR}$ response in layer 4 neurons of the sensory neocortex. Such mGluRs have also been found to be distributed in many other regions of the brain (Cartmell and Schoepp, 2000), notably in the thalamus (Reichova and Sherman, 2004), and in the hippocampus, where it may function in memory related processes (Riedel et al., 1996). Such mGluRs have been implicated in functions ranging from the control of burst/tonic firing modes of thalamic relay cells (Godwin et al., 1996a,b; Reichova and Sherman, 2004) to the gating of spike transmission in the hippocampus (Hölscher, 2002) to development of the synapse in the cerebellum (Kano et al., 2008), but it is as yet unknown if any of these functions are also subserved by the layer 4 cortical mGluRs.

Previous work has demonstrated that the layer 6 to layer 4 pathway also exhibits small, graded EPSPs and a facilitating response to paired-pulse stimulation (Stratford et al., 1996; Tarczy Hornoch et al., 1999), and was also observed for 12 cells of this study (not shown here but see Lee and Sherman, 2008). This contrasts with the synaptic properties of other inputs to layer 4 , in particular those from thalamic sources, which instead exhibit large EPSPs, a depressing response to paired-pulse stimulation, and no mGluR response (Gil et al., 1999; Lee and Sherman, 2008; MacLean et al., 2006; Rose and Metherate, 2001). Interestingly, these synaptic properties have also been found in the thalamus (Bartlett and Smith, 2002; Li et al., 2003; Reichova and Sherman, 2004), associated with two distinct classes on inputs termed "drivers" and "modulators" (Figure 3).

In the thalamus, the driver pathways, such as the retinogeniculate or layer 5 corticothalamic input (Sherman and Guillery, 2005), are the principal conduits for information flow, while the modulator pathways, such as the layer 6 corticothalamic input (Reichova and Sherman, 2004), act instead to modify information transfer through thalamus. The finding of a mGluR response in the layer 6 to layer 4 pathway, in addition to previously reported modulator properties such as paired-pulse facilitation (Stratford et al., 1996;
Tarczy Hornoch et al., 1999), suggests that this pathway also acts as a modulator (Raizada and Grossberg, 2003) (Figure 3). In this regard, the layer 6 corticothalamic neurons represent an intriguing source of divergent modulation, with separate branches potentially modulating both the thalamocortical relay cells and their layer 4 targets.

Functionally, these results suggest that the layer 6 inputs are not principally involved in the construction of receptive field properties in layer 4 of the neocortex, which instead inhere from the ascending thalamic input (Figure 3). In this respect, anatomical projection size appears to be inversely correlated with functional weight, since thalamic inputs comprise roughly $5 \%$ of cortical synapses (Binzegger et al., 2004; Latawiec et al., 2000; Lee et al., 2004), while the intracortical inputs from layer 6 contribute about $45 \%$ (Ahmed et al., 1994; Binzegger et al., 2004). Such an inverse functional relationship may represent a general principle of forebrain organization, since inputs to the thalamus exhibit a similar pattern, e.g. in the visual thalamus, retinogeniculate driver inputs comprise $5 \%$ of thalamic synapses, while layer 6 corticothalamic modulator inputs contribute about 30\% (Sherman and Guillery, 2002).

Finally, these results suggest that many of the "driver" and "modulator" criteria extend to cortical synapses and may thus serve as a useful physiological metric to trace the flow of information throughout the forebrain, although it is possible that not all pathways will be as distinctly parsed as those described thus far. Several anatomical criteria have also been shown to correlate with these physiological properties in the thalamus, such as the dense terminal arbors and proximal synapses of driver synapses (Figure 3), which also appear to be present in neocortical synapses (Ahmed et al., 1994; Huang and Winer, 2000; Latawiec et al., 2000), and it will be of interest to determine their role in further characterizing driver and modulator synapses in the neocortex (Binzegger et al., 2004).

\section{ACKNOWLEDGMENTS}

We thank Y. Lam and C. Varela for their experimental advice and J. S. Roseman and C. S. Nelson for their assistance. This work was supported by NIH Grants R01EY003038, R01DC008794 (SMS) and F32NS054478 (CCL). 


\section{REFERENCES}

Ahmed, B., Anderson, J. C., Douglas, R. J., Martin, K. A., and Nelson J. C. (1994). Polyneuronal innervation of spiny stellate neurons in cat visual cortex. J. Comp. Neurol. 341, 39-49.

Bartlett, E. L., and Smith, P. H. (2002). Effects of paired-pulse and repetitive stimulation on neurons in the rat medial geniculate body. Neuroscience 113, 957-974.

Binzegger, T., Douglas, R. J., and Martin, K. A. (2004). A quantitative map of the circuit of cat primary visual cortex. J. Neurosci. 24, 8441-8453.

Cartmell, J., and Schoepp, D. D. (2000). Regulation of neurotransmitter release by metabotropic glutamate receptors. J. Neurochem. 75, 889-907.

Ferster, D., Chung, S., and Wheat, H. (1996). Orientation selectivity of thalamic input to simple cells of cat visual cortex. Nature 380, 249-252.

Gil, Z., Connors, B. W., and Amitai, Y. (1999). Efficacy of thalamocortical synaptic connections: quanta, innervation, and reliability. Neuron 23, 385-397.

Godwin, D. W., Van Horn, S. C., Erişir, A., Sesma, M., Romano, C., and Sherman, S. M. (1996a). Ultrastructural localization suggests that retinal and cortical inputs access different metabotropic glutamate receptors in the lateral geniculate nucleus. J. Neurosci. 16, 8181-8192.

Godwin, D. W., Vaughan, J. W., and Sherman, S.M. (1996b). Metabotropic glutamate receptors switch visual response mode of lateral geniculate nucleus cells from burst to tonic. J. Neurophysiol. 76, 1800-1816.

Hirsch, J. A., and Martinez, L. M. (2006). Circuits that build visual cortical receptive fields. Trends Neurosci. 29, 30-39.

Hölscher, C. (2002). Metabotropic glutamate receptors control gating of spike transmission in the hippocampus area CA1. Pharmacol. Biochem. Behav. 73, 307-316.
Huang, C. L., and Winer, J. A. (2000). Auditory thalamocortical projections in the cat: Laminar and areal patterns of input. J. Comp. Neurol. 427, 302-331.

Kano, M., Hashimoto, K., and Tabata, T. (2008). Type-1 metabotropic glutamate receptor in cerebellar Purkinje cells: a key molecule responsible for long-term depression, endocannabinoid signalling and synapse elimination. Philos. Trans. R. Soc. Lond. B Biol. Sci. 363, 2173-2186.

Lam, Y. W., and Sherman, S. M. (2007) Different topography of the reticulothalamic inputs to first- and higher-order somatosensory thalamic relays revealed using photostimulation. J. Neurophysiol. 98, 2903-2909.

Latawiec, D., Martin K. A., and Meskenaite, V. (2000). Termination of the geniculocortical projection in the striate cortex of the monkey: a quantitative immunoelectron microscope study. J. Comp. Neurol. 419, 306-319.

Lee, C. C., Imaizumi, K., Schreriner, C. E., and Winer, J. A. (2004). Concurrent tonotopic processing streams in auditory cortex. Cereb. Cortex 14, 441-451.

Lee, C. C., and Sherman, S. M. (2008). Synaptic properties of thalamic and intracortical inputs to layer 4 of the first and higher order cortical areas in the auditory and somatosensory systems. J. Neurophysiol. 100, 317-326.

Lee, C. C., and Sherman, S. M. (2009). Glutamatergic inhibition in sensory neocortex. Cereb. Cortex (in press).

Li, J., Guido, W., and Bickford, M. E. (2003). Two distinct types of corticothalamic EPSPs and their contributions to short-term synaptic plasticity. J. Neurophysiol. 90, 3429-3440.

López-Bendito, G., Shigemoto, R. Fairén, A., and Luján, R. (2002). Differential distribution of group I metabotropic glutamate receptors during rat cortical development. Cereb. Cortex 12, 625-638.
MacLean, J. N., Fenstermaker, V., Watson, B. O., and Yuste, R. (2006). A visual thalamocortical slice. Nat. Methods 3, 129-134.

McCormick, D. A., and Von Krosigk, M. (1992). Corticothalamic activation modulates thalamic firing through glutamate "metabotropic" receptors. Proc. Natl. Acad. Sci. U.S.A. 89, 2774-2778.

Raizada, R. D., and Grossberg, S. (2003). Towards a theory of the laminar architecture of cerebral cortex Computational clues from the visual system. Cereb. Cortex 13, 100-113.

Reichova, I., and Sherman, S. M. (2004). Somatosensory corticothalamic projections: distinguishing drivers from modulators. J. Neurophysiol. 92 2185-2197.

Reid, R. C., and Alonso, J. M. (1995) Specificity of monosynaptic connections from thalamus to visual cortex. Nature 378, 281-284.

Riedel, G., Wetzel, W., and Reymann, K. G. (1996). Comparing the role of metabotropic glutamate receptors in longterm potentiation and in learning and memory. Prog. Neuropsychopharmacol. Biol. Psychiatry 20, 761-789.

Rockland, K. S., and Pandya, D. N. (1979) Laminar origins and terminations of cortical connections of the occipital lobe in the rhesus monkey. Brain Res. 179, 3-20.

Rose, H. J., and Metherate, R. (2001) Auditory thalamocortical transmission is reliable and temporally precise. J. Neurophysiol. 106, 331-340.

Ryo, Y., Miyawaki, A., Furuichi, T. and Mikoshiba, K. (1993). Immunohistochemical localization of metabotropic and ionotropic glutamate receptors in the mouse brain. Ann. N. Y. Acad. Sci. 707, 554-556.

Shepherd, G. M., Pologruto, T. A., and Svoboda, K. (2003). Circuit analysis of experience-dependent plasticity in the developing rat barrel cortex. Neuron 38, 277-289.

Sherman, S. M., and Guillery, R. W. (1998) On the actions that one nerve cell can have on another: distinguishing "drivers" from "modulators". Proc. Natl. Acad. Sci. U.S.A. 95, 7121-7126.

Sherman, S.M., and Guillery, R.W. (2002). The role of the thalamus in the flow of information to the cortex. Philos. Trans. R. Soc. Lond. B Biol. Sci. 357, 1695-1708.

Sherman, S. M., and Guillery, R. W. (2005). Exploring the thalamus and its role in cortical function. Cambridge, MIT Press.

Stinehelfer,S., Vruwink, M., and Burette, A. (2000). Immunolocalization of mGluR $1 \alpha$ in specific populations of local circuit neurons in the cerebral cortex. Brain Res. 861, 37-44.

Stratford, K. J., Tarczy-Hornoch, K., Martin, K. A. C., Bannister, N. J., and Jack, J. J. B. (1996). Excitatory synaptic inputs to spiny stellate cells in cat visual cortex. Nature 382 258-261.

Tarczy Hornoch, K., Martin, K. A. C., Stratford, K. J., and Jack, J. J. B. (1999). Intracortical excitation of spiny neurons in layer 4 of cat striate cortex in vitro. Cereb. Cortex 9, 833-843.

Conflict of Interest Statement: The authors declare that the research was conducted in the absence of any commercial or financial relationships that could be construed as a potential conflict of interest.

Received: 11 December 2008; paper pending published: 20 January 2009; accepted: 18 February 2009; published online: 26 February 2009.

Citation: Lee CC and Sherman SM (2009) Modulator property of the intrinsic cortical projection from layer 6 to layer 4. Front. Syst. Neurosci. (2009) 3:3. doi: 10.3389/neuro.06.003.2009

Copyright (c) 2009 Lee and Sherman. This is an open-access article subject to an exclusive license agreement between the authors and the Frontiers Research Foundation, which permits unrestricted use, distribution, and reproduction in any medium, provided the original authors and source are credited. 